\title{
EEG-based Upper-Alpha Neurofeedback for Cognitive Enhancement in Major Depressive Disorder: A preliminary, uncontrolled study
}

\author{
C. Escolano
}

M. Navarro-Gil

\author{
J. Minguez
}

\begin{abstract}
Conditioning of the upper-alpha rhythm to improve cognitive performance in healthy users by means of neurofeedback (NF) has been evaluated by several studies, however its effectiveness in people with severe cognitive deficits, such as depressive subjects, remains underexplored. This paper reports on a preliminary uncontrolled study to assess the effects of an upper-alpha NF intervention on patients with major depressive disorder (MDD). The NF effects on the EEG and cognitive performance were assessed. The EEG results showed that patients were able to modulate the upper-alpha rhythm in task-related EEG and during training, in both cases across the executions of the NF sessions, and pre and post within each session. The behavioral results showed the effectiveness of this intervention in a variety of cognitive functions such as working memory, attention, and executive functions.
\end{abstract}

\section{INTRODUCTION}

Major depressive disorder (MDD) is one of the most common mental illnesses, considered to be the condition responsible for most health deterioration worldwide. MDD is a mood disorder that heavily affects the quality of life of people who suffer from it, presenting a high risk of prevalence throughout life [1]. MDD is characterized, among other symptoms, by a severe cognitive impairment. The current standard treatment is antidepressant medication and psychotherapy (including cognitive, interpersonal, acceptance and commitment therapy, among others). Although these treatments generally provide symptom relief, those patients who recover but discontinue treatment have a risk of recurrence. A promising technique that has been recently explored is neurofeedback [1].

Neurofeedback (NF) is a technique that allows users to endogenously modulate their brain rhythms, using an operant conditioning paradigm. The basic principle of NF consists of measuring the brain activity (commonly via electroencephalogram, EEG), processing the brain patterns of interest, and then providing the user with feedback stimuli depending on the desired working levels of these rhythms. Several EEG-based NF studies have reported therapeutical effects in a variety of neurological and psychological disorders such as epilepsy, attention deficit hyperactivity disorder (ADHD), and addictive disorders, among others (see [2], [3] for reviews). Other studies have reported the enhancement

Carlos Escolano and Javier Mínguez are with the Instituto de Investigación en Ingeniería de Aragón (I3A) and the University of Zaragoza, Spain. Javier Minguez is also with Bit\&Brain Technologies SL, Spain. E-mail: \{cescolan, jminguez\}@unizar.es. Mayte Navarro-Gil is with Bit\&Brain Technologies SL, Spain. E-mail: maytenavarro@bitbrain.es. Javier GarcíaCampayo is with the Department of Psychiatry and Miguel Servet University Hospital, University of Zaragoza, Spain. E-mail: jgarcamp@ gmail.com. This work was supported by projects HYPER-CSD2009-00067 and DPI200914732-C02-01 of the Spanish Government, and by DGA-FSE (T04 group). of cognitive abilities in healthy users [4]-[7]. The latter studies targeted the enhancement of the upper section of the alpha band, referred to as upper-alpha band, which has been traditionally linked to performance [8].

Alpha is the dominant rhythm in the human EEG, which is characterized by a 'peak' in the power spectra in the frequency range $[7,13] \mathrm{Hz}$ (denoted alpha band), and is related to cognitive factors, such as working memory [9]. Regarding the alpha rhythm electrophysiology in depressive patients, a prefrontal alpha asymmetry has been commonly reported (see [10] for a review). While recent research studies are still elucidating whether this asymmetry is an endophenotype for depression [11], some studies have performed NF interventions to reduce such asymmetry in an attempt to reduce the depressive symptomatology [12], [13]. The results obtained, although encouraging, represent uncontrolled case series and thus controlled research is required to better evaluate its effectiveness [13]. To the best of the authors' knowledge, only one study performed a clinical trial reporting a reduction in depression while improving executive function [14]. In addition to the little evidence accumulated to date, the rationale behind that intervention is still unclear. This is because other studies have found that changes in frontal asymmetry across measurement occasions are not related to the clinical status of the patient [15], [16].

An alternative NF intervention for depression could be to perform an intervention for cognitive enhancement, which has been extensively explored in healthy users [4]-[7]. This NF intervention could alleviate the severe cognitive impairment caused by depression. In the same line, this paper reports on a preliminary uncontrolled study to assess the effectiveness of an upper-alpha NF intervention directed to improve cognitive performance in people with MDD.

\section{Methods}

\section{A. Participants and Experimental Procedure}

The sample consisted of 49 participants diagnosed with MDD (mean age: $54.08 \pm 3.28$ ). Depressive symptomatology was evaluated in an intake interview. The inclusion criteria were age range (18-65), understanding of Spanish language, diagnosis of MDD according to the Diagnostic and Statistical Manual of Mental Disorders (DSM-IV) [17], stable pharmacological and psychological treatment, and signature of the informed consent forms. The exclusion criteria were diagnosis of comorbid disorders or severe psychiatric disorders (e.g., schizophrenia, paranoia, drug addiction, dementia), and inability to perform the NF intervention. The study followed the Helsinki Convention norms and subsequent 




Fig. 1. Experimental procedure of the study. Cognitive assessments were executed pre and post the NF intervention, which consisted of $8 \mathrm{NF}$ sessions. Each NF session contained 5 NF trials and pre and post EEG screenings.

modifications, as well as the Declaration of Madrid of the World Psychiatric Association. The experimental procedure was approved by the Ethical Review Board of the regional health authority.

The experimental procedure consisted of a preliminary uncontrolled study (Figure 1). Cognitive assessments were carried out before and after the NF intervention to evaluate cognitive performance. Each participant performed $8 \mathrm{NF}$ sessions for 4 weeks on alternate days ( 2 sessions per week). Each NF session consisted of 5 training trials (20 min), with pre and post EEG screenings to assess EEG changes.

\section{B. Cognitive Assessments}

The pre and post cognitive assessments lasted approximately 30 minutes and comprised five psychological tests: $(i)$ Paced Auditory Serial Addition Task (PASAT) [18] evaluates the rate of information processing and working memory. This test is sensitive to minimal changes in neurocognitive performance and presents high levels of internal consistency and test-retest reliability [19]. The test scores were the number of errors and performance time. (ii) Rey Auditory Verbal Learning Test (RAVLT) [20] (Spanish version [21]) evaluates the retention and immediate evocation, verbal learning, and recognition [22]. The test scores were the number of correct and the number of recognized words. (iii) Stroop ColorWord Test (STROOP) [23] evaluates cognitive performance, attention, concentration, and measures the individual capacity to solve cognitive stress, inhibit interferences and process complex data [21]. The test scores were the performance in a task consisting of reading the names of colors (Word) and naming the ink color (Color), performance in an incongruent task (Color-Word), and interference. (iv) Trail Making Test (TMT) [24] evaluates the information on visual search, scanning, speed of processing, mental flexibility and executive functions [25]. This test consists of two parts: TMT-A evaluates attention and concentration and TMT-B measures executive functions, such as planning and mental flexibility. The score was the time elapsed to complete each part of the test. $(v)$ Fluency Verbal Test (FAS) [26] evaluates verbal phonetic fluency. The test score was the number of evoked words. Statistical significance was assessed using non-parametric Wilcoxon tests for paired samples.

\section{EEG Data Collection and Neurofeedback Intervention}

EEG was recorded during the EEG screenings and NF training trials. 16 EEG sensors were placed at FP1, FP2, F3, $\mathrm{Fz}, \mathrm{F} 4, \mathrm{C} 3, \mathrm{Cz}, \mathrm{C} 4, \mathrm{P} 7, \mathrm{P} 3, \mathrm{Pz}, \mathrm{P} 4, \mathrm{P} 8, \mathrm{O} 1, \mathrm{Oz}$ and $\mathrm{O} 2$ (subset of the 10/10 system), with the ground and reference electrodes on $\mathrm{FPz}$ and on the left earlobe, respectively. EEG was amplified and digitized using a gTec system at a sampling rate of $256 \mathrm{~Hz}$, power-line notch-filtered at 50 $\mathrm{Hz}$ and $[0.5,60] \mathrm{Hz}$ bandpass-filtered. Signal acquisition, processing and feedback presentation were developed using Bit\&Brain Technologies software.

1) EEG Screenings: The screenings were carried out pre and post the NF training in each session. Each screening comprised a basal EEG and a task-related EEG data collection. The basal screening lasted $3 \mathrm{~min}$ in a closed-eyes resting state. The task-related screening lasted $3 \mathrm{~min}$ in an open-eyes active state: participants faced a square on a computer screen that changed randomly from gray to red or from gray to blue gradually (i.e., changing saturation color), and they had to count the number of saturation changes from gray to red as a cognitive challenge [5].

2) Neurofeedback Training: The NF training focused on the enhancement of upper alpha (UA) power in the parietooccipital (PO) area of the scalp. Each session comprised the execution of $5 \mathrm{NF}$ training trials (4 min each), and consisted of two steps: $(i)$ calibration to individualize the training to each user, and $(i i)$ online training. In both steps, the EEG power was calculated with a sliding window FFT with a 1 sec hamming window, $30 \mathrm{msec}$ of overlapping, and 1024 points using zero-padding $(0.25 \mathrm{~Hz}$ resolution).

In the calibration step, the EEG was firstly filtered from artifacts. An Independent Component Analysis (ICA) filter was computed on the pre-NF task-related EEG screening, where the blinking component was automatically detected (the component with the most number of samples higher than $75 \mu \mathrm{V}$ in prefrontal sensors) and removed. The UA frequency band was defined as the frequency interval [IAF, IAF+2] $\mathrm{Hz}$, where the Individual Alpha Frequency (IAF) was the frequency bin with the maximum power value in the alpha range $([7,13] \mathrm{Hz})[8]$. The IAF was computed per sensor on the power spectra of the pre-NF task-related EEG screening, and when no clear alpha peak was found the power spectra of the basal EEG screening was used instead. Then, the UA power was computed for the sensors P3, Pz, P4, O1 and O2 (referred to as feedback sensors) to set the online training. The distributions of UA power values were computed for the feedback sensors, and then averaged across them. The lower and upper limits of the resulting distribution ( $5 t h, 95 t h$ percentiles) and the baseline (mean value) were used to normalize the UA power values during the online training. During the online training, the feedback was visually provided by a square that changed its saturation color on a computer screen. The saturation was proportional to the deviation of the UA power from the baseline (saturation scale covered the lower and upper limits of the power distribution), and updated every $30 \mathrm{msec}$. 

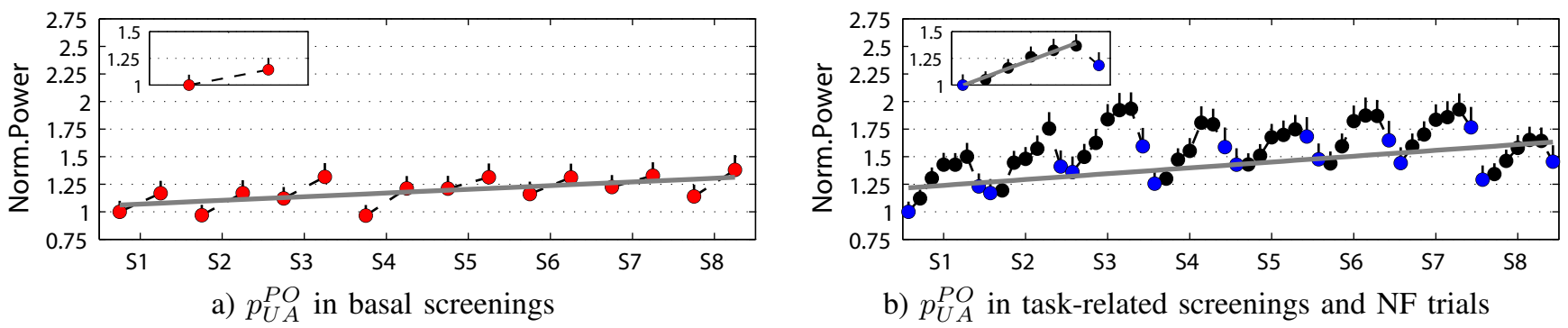

Fig. 2. UA power for the feedback sensors $\left(p_{U A}^{P O}\right)$ in the EEG screenings and NF trials for each session. Red and blue dots denote basal and task-related screenings, respectively, and black dots denote trials. Values are normalized per user to $p_{U A}^{P O}$ in the pre-NF screening of first session. The grey line shows the across-session tendency of the pre-NF screenings. The upper-left boxes show the within-session values, which are normalized per user to $p_{U A}^{P O}$ in the pre-NF screening. The grey line shows the within-session tendency of the pre-NF screening and the five NF trials. Vertical bars display SEM.

\section{EEG Analysis}

Artifact filtering procedure consisted of three steps: $(i)$ filtering the blinking artifacts using ICA, (ii) a threshold filtering in the time domain to eliminate high amplitude epochs, and ( $\mathrm{iii}$ ) a z-score filtering in the frequency domain to eliminate prominent EOG and EMG activity. Firstly, for the EEG data of each NF session the blinking component of the ICA decomposition was identified by visual inspection, and its consistency across sessions was statistically checked [27]. Then, the EEG data of each session was reconstructed removing this component. Secondly, the resulting EEG data was divided into $1 \mathrm{sec}$ epochs and those epochs with samples presenting an absolute amplitude higher than $150 \mu \mathrm{V}$ were removed. Finally, z-scores were computed for each sensor in the frequency bands $[0,4] \mathrm{Hz}$ and $[20,30] \mathrm{Hz}$ (commonly affected by EOG and EMG artifacts, respectively), and those epochs with a $\mathrm{z}$-score value higher than 2 were removed.

The effects of the NF were assessed on the UA power for the feedback sensors (parieto-occipital area, PO), denoted as $p_{U A}^{P O}$, using three metrics: $(i)$ basal progress evaluated the effects on the resting state, measured in the basal screenings; ( ii) task-related progress evaluated the enhancement degree on a normalized task, measured in the task-related screenings; and (iii) training progress measured the enhancement degree during training in comparison to the baseline. These three metrics measured both across and within sessions effects (i.e., averaging the EEG data for all the sessions). Across sessions, metrics $(i)$ and $(i i)$ were measured as the (Pre, Post) $p_{U A}^{P O}$ comparison of the pre-NF screening of session 1 vs session 8 , and as the $p_{U A}^{P O}$ tendency of the preNF screenings of all sessions; within sessions, they were measured as the (Pre, Post) $p_{U A}^{P O}$ comparison of the pre vs post screening. Across sessions, metric (iii) was measured as the (Pre, Post) $p_{U A}^{P O}$ comparison of the pre-NF taskrelated screening of session 1 vs trial 5 of session 8; and within sessions, it was measured as the (Pre, Post) $p_{U A}^{P O}$ comparison of the pre-NF task-related screening vs trial 5, and as the $p_{U A}^{P O}$ tendency of the pre-NF task-related screening and the five trials. (Post/Pre) ratios were reported for the (Pre, Post) comparisons, and tendency was computed as the gradients of a fitted regression line. Statistical significance was assessed using Wilcoxon tests for paired samples.

\section{RESULTS}

3 participants were excluded from EEG and behavioral analyses ( 1 did not perform the cognitive assessments and 2 were not able to complete the NF intervention due to visual fatigue). The final sample consisted of 46 participants.

\section{A. EEG Analysis}

Figure 2 shows the UA power for the feedback sensors $\left(p_{U A}^{P O}\right)$ in the EEG screenings and NF training trials of all the sessions. Results are normalized per user to the $p_{U A}^{P O}$ in the pre-NF screening of the first session due to the large interuser variability in absolute power. Pre-post $p_{U A}^{P O}$ in basal EEG was not significantly increased across sessions $(17.74 \%, p=$ $0.15)$, whereas it was significant within sessions $(13.62 \%$, $p=0.049)$. Pre-post $p_{U A}^{P O}$ in task-related EEG was significantly enhanced across sessions $(45.79 \%, p<0.001)$ and within sessions $(15.82 \%, p<0.001)$. The tendency values across sessions in both metrics were significantly higher than zero: 0.013 for the basal EEG $(p<0.001)$ and 0.021 for the task-related EEG $(p<0.001)$. Training progress metric was significant across sessions $(88.79 \%, p<0.001)$ and within sessions $(33.9 \%, p<0.001)$. In addition, the withinsession tendency was 0.074 , significantly higher than zero $(p<0.001)$. The higher enhancement ratios were found in task-related EEG and during training.

In summary, the results suggest that the participants were able to learn how to modulate the UA power in the parietooccipital area of the scalp, thus showing a clear increase in the task-related screenings and NF trials across and within sessions, with the training effects remaining over sessions (each session was built upon the previous one). In addition, this intervention also increased the UA power in the basal EEG in a closed-eyes state to a lesser extent.

\section{B. Cognitive Assessments}

Mean and SEM scores for the pre and post executions of the psychological tests are displayed in Table I, with the significance levels. PASAT evaluated attention and working memory, with the latter being related to alpha rhythm [28]. Both the number of errors and time elapsed were significantly improved. RAVLT evaluated verbal memory. A significant increase was found in the number of correct and recognized words. TMT evaluated executive functions, showing 
TABLE I

SCORES ON THE Cognitive Assessments

\begin{tabular}{llccc}
\hline Test & Scores & Pre & Post & p-value \\
\hline \multirow{2}{*}{ PASAT } & Errors & $13.60(1.11)$ & $9.54(1.01)$ & $<\mathbf{0 . 0 0 1}$ \\
& Time & $247.54(12.35)$ & $208.77(11.08)$ & $<\mathbf{0 . 0 0 1}$ \\
\hline \multirow{2}{*}{ RAVLT } & Correct words & $52.85(2.18)$ & $60.74(2.24)$ & $<\mathbf{0 . 0 0 1}$ \\
& Recogn. words & $11.96(0.42)$ & $13.04(0.27)$ & $<\mathbf{0 . 0 0 1}$ \\
\hline \multirow{3}{*}{ STROOP } & Word & $52.91(1.83)$ & $56.09(1.92)$ & $<\mathbf{0 . 0 0 1}$ \\
& Color & $42.78(1.36)$ & $46.00(1.51)$ & $<\mathbf{0 . 0 0 1}$ \\
& Color-Word & $47.02(1.79)$ & $51.78(1.78)$ & $<\mathbf{0 . 0 0 1}$ \\
& Interference & $51.69(1.10)$ & $53.33(0.92)$ & 0.111 \\
\hline \multirow{2}{*}{ TMT } & TMT-A time & $45.84(4.04)$ & $39.45(3.97)$ & $<\mathbf{0 . 0 0 1}$ \\
& TMT-B time & $111.07(16.71)$ & $87.68(12.70)$ & $\mathbf{0 . 0 0 3}$ \\
\hline \multirow{2}{*}{ FAS } & Num. letters & $43.27(1.85)$ & $46.00(1.88)$ & $\mathbf{0 . 0 0 9}$ \\
\hline
\end{tabular}

an improvement in both parts of the test. STROOP evaluated attention and concentration. The word, color and color-word scores were significantly improved, showing an increase in concentration. The interference score was improved but with no statistical significance $(p=0.111)$. FAS evaluated verbal fluency, showing a significant improvement.

In summary, these results show a general improvement in cognitive performance after the NF intervention targeting functions such as working memory, attention, concentration, verbal memory, verbal fluency, and executive functions.

\section{CONCLUSiOnS}

A NF intervention in the upper-alpha band and parietooccipital area of the scalp has already demonstrated to improve performance in healthy users [4]-[7]. To the authors' knowledge, the current study is one of the first research studies assessing the effectiveness of such an intervention to improve the cognitive performance in patients with major depressive disorder (MDD). The results show that 46 depressive patients were able to significantly enhance the upperalpha rhythm in task-related EEG and during training, both across and within the executions of the NF sessions. Basal EEG was enhanced to a lesser extent. The psychological tests PASAT, RAVLT, TMT and FAS were improved (and partially the STROOP), which shows the effectiveness of this intervention in a variety of cognitive functions such as working memory, attention, concentration, verbal memory, verbal fluency, and executive functions. However, these results must be interpreted with caution as this is an open-label uncontrolled study, where motivation of the participants and practice effects were not controlled. Based on the positive findings of this preliminary study, a randomized controlled trial is the object of future work.

\section{REFERENCES}

[1] R.J. DeRubeis, G.J. Siegle, and S.D. Hollon, "Cognitive therapy versus medication for depression: treatment outcomes and neural mechanisms," Nat. Rev. Neurosci., vol. 9, no. 10, pp. 788-796, 2008.

[2] E. Basar and B. Güntekin, "A review of brain oscillations in cognitive disorders and the role of neurotransmitters.," Brain research, vol. 1235, pp. 172-93, 2008.

[3] S. Niv, "Clinical efficacy and potential mechanisms of neurofeedback," Pers Indiv Differ, vol. 54, no. 6, pp. 676 - 686, 2013.
[4] C. Escolano, M. Aguilar, and J. Minguez, "EEG-based upper alpha neurofeedback training improves working memory performance," in Proc. Int. Conf. IEEE EMBC, 2011, pp. 2327-2330.

[5] B. Zoefel, R. J. Huster, and C. S. Herrmann, "Neurofeedback training of the upper alpha frequency band in EEG improves cognitive performance," NeuroImage, vol. 54, no. 2, pp. 1427 - 1431, 2011.

[6] S. Hanslmayr, P. Sauseng, M. Doppelmayr, M. Schabus, and W. Klimesch, "Increasing individual upper alpha power by neurofeedback improves cognitive performance in human subjects," Applied Psychophysiology and Biofeedback, vol. 30, pp. 1-10, 2005.

[7] M. V. Alexeeva, N. V. Balios, K. B. Muravlyova, E. V. Sapina, and O. M. Bazanova, "Training for voluntarily increasing individual upper alpha power as a method for cognitive enhancement," Human Physiology, vol. 38, no. 1, pp. 40-48, 2012.

[8] W. Klimesch, "EEG alpha and theta oscillations reflect cognitive and memory performance: a review and analysis," Brain Research Reviews, vol. 29, no. 2-3, pp. $169-195,1999$.

[9] P. Sauseng, W. Klimesch, K. F. Heise, W. R. Gruber, E. Holz, A. Karim, M. Glennon, C. Gerloff, N. Birbaumer, and F. C. Hummel, "Brain oscillatory substrates of visual short-term memory capacity," Current biology, vol. 19, no. 21, pp. 1846-52, 2009.

[10] J.A. Coan and J.J. Allen, "Frontal EEG asymmetry as a moderator and mediator of emotion," Biol psychol, vol. 67, no. 1, pp. 7-50, 2004.

[11] J.J. Allen and M.X. Cohen, "Deconstructing the "resting" state: exploring the temporal dynamics of frontal alpha asymmetry as an endophenotype for depression," Frontiers in Human Neuroscience, vol. 4, pp. 232, 2010.

[12] E. Baehr, J.P Rosenfeld, and R. Baehr, "The clinical use of an alpha asymmetry protocol in the neurofeedback treatment of depression," Journal of Neurotherapy, vol. 2, no. 3, pp. 10-23, 1997.

[13] D.C. Hammond, "Neurofeedback treatment of depression and anxiety," Journal of Adult Development, vol. 12, no. 2, pp. 131-137, 2005.

[14] S.W. Choi, S.E. Chi, S.Y. Chung, J.W. Kim, C.Y. Ahn, and H.T. Kim, "Is alpha wave neurofeedback effective with randomized clinical trials in depression? A pilot study," Neuropsychobiology, vol. 63, no. 1, pp. 43-51, 2011

[15] S. Debener, A. Beauducel, D. Nessler, B. Brocke, H. Heilemann, and J. Kayser, "Is resting anterior EEG alpha asymmetry a trait marker for depression?", Neuropsychobiology, vol. 41, no. 1, pp. 31-37, 2000.

[16] J.J. Allen, H.L. Urry, S.K. Hitt, and J.A. Coan, "The stability of resting frontal electroencephalographic asymmetry in depression," Psychophysiology, vol. 41, no. 2, pp. 269-280, 2004.

[17] American Psychiatric Association, Diagnostic criteria from DSM-IV, American Psychiatric Publishing Incorporated, 1994.

[18] D. M. A Gronwall, "Paced auditory serial-addition task: a measure of recovery from concussion," Perceptual and motor skills, vol. 44, no. 2, pp. 367-373, 1977.

[19] T. N. Tombaugh, "A comprehensive review of the Paced Auditory Serial Addition Test (PASAT)," Archives of Clinical Neuropsychology, vol. 21, no. 1, pp. 53-76, 2006.

[20] A. Rey, L'examen clinique en psychologie., Presses Universitaires de France, 1958.

[21] M. D. Lezak, D. B. Howieson, and D. W. Loring, Neuropsychological assessment, Oxford University Press, New York, 2004.

[22] J. P. Miranda and R. R. Valencia, "English and Spanish versions of a memory test: Word-length effects versus spoken-duration effects," Hispanic J. of Behavioral Sciences, vol. 19, no. 2, pp. 171-181, 1997.

[23] J. R. Stroop, "Studies of interference in serial verbal reactions.," J. of Experimental Psychology: General, vol. 121, no. 1, pp. 15, 1992.

[24] R. M. Reitan, "Validity of the Trail Making Test as an indicator of organic brain damage," Perceptual and motor skills, vol. 8, no. 3, pp. 271-276, 1958

[25] T. N. Tombaugh, "Trail Making Test A and B: normative data stratified by age and education," Archives of Clinical Neuropsychology, vol. 19, no. 2, pp. 203, 2004.

[26] A. L. Benton and K. Hamsher, "Multilingual aphasia examination," Iowa City: University of Iowa, 1976.

[27] A. Hyvärinen, "Testing the ICA mixing matrix based on inter-subject or inter-session consistency.," Neurolmage, vol. 58, no. 1, pp. 122-36, 2011.

[28] W. Klimesch, P. Sauseng, and S. Hanslmayr, "EEG alpha oscillations: the inhibition-timing hypothesis.," Brain research reviews, vol. 53, no. 1, pp. 63-88, 2007. 